\title{
Après le diplôme. Les parcours migratoires au sortir des hautes écoles
}

\author{
M. Gauthier \\ Observatoire Jeunes et Société, Institut national de la recherche scientifique, 490, rue de la Couronne, \\ Québec, G1K 9A9, Canada \\ Correspondence to: M. Gauthier (madeleine_gauthier@ucs.inrs.ca)
}

Rérat, P.: Après le diplôme. Les parcours migratoires au sortir des hautes écoles, Neuchâtel, Éditions Alphil-Presses universitaires suisses, 294 pp., ISBN-13: 978-2-940489-367, €27, 2013.

Voilà une étude qui fera certainement école par son propos mais aussi par sa rigueur d'analyse et son originalité. La question de recherche à l'origine de ce volume concerne le parcours migratoire des jeunes diplômés du canton du Jura en Suisse. Elle se pose d'abord en termes d' «exode des cerveaux» observé par le solde migratoire des communes les plus rurales et les plus périphériques du canton par rapport à celles qui sont plus urbanisées. L'étude ne tient pas compte cependant d'une possible immigration en provenance d'autres cantons ou d'ailleurs et qui aurait pu atténuer l'effet de l'émigration des plus scolarisés. Le volume offre d'abord une abondante revue de littérature faisant état de la recherche sur le déséquilibre que le phénomène de la migration des plus scolarisés peut causer. Il en est de même des différentes théories qui concernent le phénomène migratoire. L'exercice sera d'une grande utilité pour qui voudrait entreprendre une étude sur le même sujet.

L'auteur, pour sa part, s'est orienté vers une approche sociologique qui conçoit la question de la migration comme «la résultante d'un choix sous contrainte» (p. 35), la marge de manœuvre variant entre plusieurs contraintes. Ce choix lui apparaissait important parce que centré sur l'acteur. Il devait conduire non pas à un bilan démographique ou à la recherche du facteur dominant, mais à la connaissance du profil des acteurs en question, de leurs parcours migratoires et des arbitrages qui les sous-tendent, de leurs motivations, leurs projets et leur attachement au canton. Cette perspective permet de mieux évaluer le poids de la contrainte économique trop facilement identifiée dans l'opinion publique comme la seule à l'origine de ce qui s'est longtemps décrit comme un «exode» ce qui, par définition, ne peut présager d'un retour.
Ce choix orientait l'auteur vers une étude de cas à partir d'un questionnaire. Il a constitué un échantillon de 924 répondants comprenant des diplômés de différentes écoles entre 2000 et 2010: hautes écoles universitaires (HEU), hautes écoles pédagogiques (HEP) et hautes écoles spécialisées (HES) de même que des étudiants. Ils ont été repérés par divers moyens dont le bouche-à-oreille à l'intérieur et à l'extérieur du canton dans le but de les inviter ultérieurement à remplir un questionnaire en ligne. Cette méthode s'avère plus rapide et est devenue efficace grâce à la généralisation d'Internet.

Tout comme pour l'option théorique, la méthodologie est clairement décrite et l'analyse est exposée dans un style qui ne rebuterait pas quiconque pourrait craindre de se perdre dans les méandres de l'analyse statistique. Les tableaux illustrent bien chacune des analyses. Les derniers paragraphes font ressortir quelques limites: recherche à compléter par une démarche qualitative, critères à élargir pour la population à l'étude et cueillette à effectuer pour connaître le point de vue des employeurs jurassiens. Ces limites exprimées par l'auteur n'enlèvent rien à la rigueur de l'analyse et à la richesse des résultats. Elles ne font qu'annoncer ce que pourrait être la poursuite d'un tel projet.

Qu'en est-il du profil des répondants au questionnaire? La question migratoire concernerait davantage les diplômés universitaires que les diplômés des hautes études spécialisées ce qui s'observe aussi ailleurs, la localisation des universités et un plus grand choix de carrière pour les diplômés universitaires étant identifiés aux milieux de plus grande densité. L'accès du plus grand nombre aux études supérieures a fait en sorte, dans le Jura comme ailleurs, de multiplier les motifs de départ, mais aussi, constat à ne pas négliger, les possibilités de retour ou de se stabiliser.

L'enquête conduit à des constats où se multiplient les variables: territoriales, sociales, culturelles et personnelles. Même le facteur linguistique entre en ligne de compte pour ce 
canton francophone ce qui n'étonnera pas le lecteur québécois, les migrations interprovinciales étant moins importantes au Québec que dans les provinces anglophones. De même, le solde migratoire des communes rurales et périphériques s'inscrit-il dans le phénomène de métropolisation en Occident. Au-delà de ces tendances macrosociales, d'autres facteurs peuvent cependant favoriser la propension à demeurer ou à revenir dans le canton. L'auteur n'en reste pas à une description de premier niveau. Par l'analyse multivariée, il en arrive à identifier les facteurs qui influencent le plus la propension au retour ou à la stabilisation tout comme ceux à un non-retour.

En ce qui concerne le retour ou la stabilisation résidentielle dans le canton, en particulier pour les diplômés universitaires, des facteurs personnels comme la proximité du cercle d'amis et de la famille, l'optique de fonder une famille, l'attachement au canton, mais aussi l'opportunité professionnelle (par exemple, l'enseignement est garant de stabilité résidentielle) et le cadre de vie entrent en ligne de compte. Il existe une forte corrélation entre les facteurs influençant les choix migratoires des diplômés universitaires et des diplômés des autres secteurs d'enseignement. Pour effectuer un certain départage, le chercheur a constitué des axes de différenciation en tenant compte de «l'importance donnée par chaque diplômé aux critères proposés dans sa décision d'aller dans le Jura» (p. 157) : attachement au canton, poids relatif accordé aux aspects économiques, carrière professionnelle, famille, ce qui donne un score pour chaque axe et pour chacun des diplômés et a permis de créer une typologie. L'auteur souligne le fait que la possibilité d'un retour dans le Jura ne dépend pas «uniquement de variables liées au marché du travail et à sa structure». Les résultats, ajoutera-t-il, «donnent une première indication du fait que les motivations des diplômés dans leur comportement migratoire répondent à une pluralité de logiques» (p. 149). Ces logiques sont d'ordre personnel mais peuvent aussi être liées aux opportunités professionnelles et au cadre de vie.

Il est difficile de résumer en ces quelques mots toute la richesse du matériel recueilli lors de cette enquête. Notons toutefois, que la perspective de retour est à la fois désirable et réalisable seulement pour un diplômé universitaire sur cinq vivant à l'extérieur du Jura. Pour un autre cinquième, elle est désirable mais non réalisable. Les principaux obstacles tiennent au travail du conjoint, à ses aspirations et à ses perspectives de carrière, au cercle d'amis et à l'implication dans des activités associatives et culturelles. Les obstacles au retour n'enlèvent rien à l'attachement au Jura qui est généralement positif chez tous les répondants. Les opinions les plus négatives concernent le marché du travail, celles qui sont plutôt relatives vont aux services et aux infrastructures et les plus positives, à l'environnement social et naturel.

L'auteur de cette recherche financée par un prix de la Fondation pour la création culturelle dans le Jura pouvait-il passer outre aux possibles applications de ses résultats de recherche? Il refuse explicitement de le faire, ce rôle n'appartenant pas, selon lui, au chercheur. Il ne se dispense cependant pas, en guise de conclusion, de laisser trois messages à portée générale qui pourront nourrir les débats politiques.

Le premier message reconnaît que si les flux migratoires sont orientés vers les zones urbaines pour les diplômés, en particulier parmi les détenteurs des diplômes les plus élevés, cela va dans le sens de la littérature sur la question. Des particularités du territoire favorisent cependant ce phénomène : flux migratoires confinés dans la partie francophone de la Suisse, caractère rural de la région, localisation des hautes écoles qui oblige au déménagement. Par contre, les contreflux ne sont pas à négliger dont certains peuvent aussi être propres à la Suisse: décentralisation des structures administratives et petite taille du pays qui favorisent la proximité des centres urbains et le marché du travail.

Le caractère sélectif de la population migrante doit aussi être pris en considération: disparités selon le profil des diplômés, segmentation du marché du travail et complexification des parcours de vie et du statut familial.

Enfin, le dernier message concerne le peu de référence porté aux questions financières. L'auteur rappelle que les possibilités pour le conjoint ou la conjointe de trouver un poste de travail et les perspectives de carrière auxquelles il faut ajouter la vie sociale et culturelle constituent un ensemble de questions qu'il ne faut pas négliger. L'auteur fait cependant remarquer, à l'encontre de la thèse de R. Florida, La classe créative (1974), que l'offre culturelle n'est pas plus prédominante que la question financière mais se combine aux autres dimensions.

Les recherches contemporaines comme celle-ci ont leur mérite en ce qu'elles ont fait ressortir la diversité des logiques migratoires en partant de l'acteur. De ce fait, elles ont révélé la possible réversibilité des mobilités ce que dissimulait la notion d'exode et éloignait d'autant la perspective pour les régions ou les milieux ruraux d'agir en conséquence. Cette démarche fut aussi celle du Groupe de recherche sur la migration des jeunes au Québec qui en a apprécié les vertus.

Le livre de Patrick Rérat est à lire et à faire lire! 